\title{
Erratum
}

\section{Fresh-water lenses and practical limitations of their three-dimensional simulation}

\author{
F. Ghassemi • K. Alam • K.W.F. Howard
}

Hydrogeology Journal (2000) 8:521-537

In the printed version of the article, an error has occurred on page 526, equation (1). This equation should read

$\mathrm{R}=\mathrm{P}-\mathrm{E} \pm \mathrm{dV}$

Published online: 27 April 2001

(C) Springer-Verlag 2001

The online version of the original article can be found at http://dx.doi.org/10.1007/s100400000087

F. Ghassemi (

Centre for Resource and Environmental Studies,

Australian National University, Canberra,

Australian Capital Territory 0200, Australia

e-mail: fredg@cres.anu.edu.au

Fax: +61-2-62490757

K. Alam

ECOWISE Environmental, ACTEW Corporation,

16 Lithgow Street, Fyshwick, Australia Capital Territory 2609,

Australia

K.W.F Howard

Groundwater Research Group, University of Toronto,

1265 Military Trail, Scarborough, Ontario M1C 1A4,

Canada 